\title{
Commentary: RAGE against the spleen
}

\author{
Richa Dhawan, MD, MPH, and Mark A. Chaney, MD
}

From the Department of Anesthesia and Critical Care, University of Chicago Medical Center, Chicago, Ill. Disclosures: Authors have nothing to disclose with regard to commercial support.

Received for publication Sept 27, 2018; accepted for publication Sept 28, 2018; available ahead of print Oct 30, 2018.

Address for reprints: Richa Dhawan, MD, MPH, Department of Anesthesia and Critical Care, University of Chicago Medical Center, 5841 S Maryland Ave, MC 4028, Chicago, IL 60637 (E-mail: rdhawan@ dacc.uchicago. edu).

J Thorac Cardiovasc Surg 2019;157:2272-3

$0022-5223 / \$ 36.00$

Copyright (C) 2018 Published by Elsevier Inc. on behalf of The American Association for Thoracic Surgery

https://doi.org/10.1016/j.jtcvs.2018.09.071

In their remarkable animal experiment described in this issue of the Journal, Tian and colleagues ${ }^{1}$ elucidate the pathway of myocardial reperfusion injury (RI) and demonstrate an intervention to counteract the chain reaction. Tian and colleagues ${ }^{1}$ induced myocardial ischemia in mice by left coronary occlusion and found that this resulted in higher levels of high-mobility group box 1 protein (HMGB1) and cell-free DNA (cfDNA) in the myocardium and plasma. Treatment with either deoxyribonuclease 1 or anti-HMGB1 antibodies significantly decreased the infarction size. Additionally, administering HMGB1 and cfDNA (exogenous ischemic cardiac coronary perfusate) during reperfusion significantly increased infarction size. Splenectomized, RAGE-knockout, and toll-like receptor 9 (TLR9)knockout mice attained cardioprotection from RI, presumably from inhibition of the inflammatory response. This suggests that HMGB1 and cfDNA contribute to ischemic RI by triggering splenic RAGE- and TLR9dependent pathways. These findings hold enormous potential for larger animal studies and eventual pharmacologic intervention in human patients.

DNA is generally organized into nucleosomes, with a small amount circulating freely in the plasma, referred to as cell-free DNA. Large quantities of cfDNA, resulting from either cell death or impaired clearance, have been known to cause havoc in the body. Extracellular DNA can be grouped among a vicious team of players referred to as damage-associated molecular patterns. When DNA becomes loose from its native structure, DNA-binding proteins such as histones and HMGB1 are also released into the circulation. Damage-associated molecular patterns are mediators of progressive chain reactions through the activation of inflammatory stimuli, such as TLR9 and RAGE. The subsequent release of splenic leukocytes then amplifies myocardial injury. ${ }^{2}$ Although cfDNA was described decades ago, there has been a remarkable surge of research in the last few years that has paved the way for the study conducted by Tian

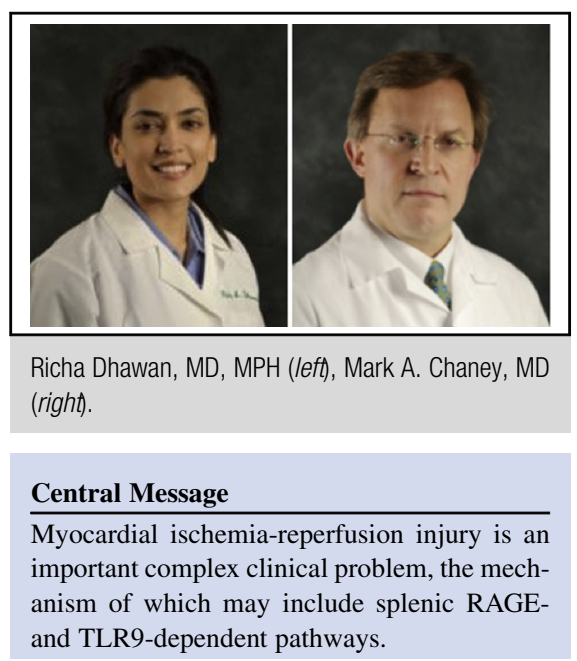

See Article page 2256.

and colleagues. ${ }^{1}$ This includes identification of specific proteins, receptors, and pathways that are involved in this axis of cellular death.

Size of infarction tissue matters, and the current mainstay of therapy in patients with ST-segment elevation myocardial infarction consists of rapid reperfusion by either thrombolysis or primary percutaneous coronary intervention. Despite novel diagnostic techniques, interventional technology, and antiplatelet drugs, morbidity and mortality after myocardial infarction remains a substantial problem. Why is it not sufficient to reperfuse ischemic tissue? Paradoxically, the very act of reperfusion leads to a chain reaction of chemical mediators that destroy myocardial cells. The RI can manifest as reperfusion arrhythmias, as myocardial stunning, or as cell death. $^{3,4}$

Tian and colleagues ${ }^{1}$ have conducted a complex, welldesigned study; however, some limitations exist. They do not provide any direct evidence of change in cardiac functional status as a result of a decrease in infarction size. Although such can be inferred from previous studies, direct measurement of improvement in function is an important primary outcome. The pathophysiology of RI is complex and not limited solely to RAGE and TLR9. This study does not identify the specific pathway in which these mediators interact. No clinically effective therapy is currently available to combat RI after STsegment elevation myocardial infarction, making this study an important early step towards translational research. 


\section{References}

1. Tian Y, Charles EJ, Yan Z, Wu D, French BA, Kron IL, et al. The myocardial infarct-exacerbating effect of cell-free DNA is mediated by the high-mobility group box 1-receptor for advanced glycation end products-Toll-like receptor 9 pathway. J Thorac Cardiovasc Surg. 2019;157: 2256-69.e3.

2. Zheng Y, Gardner SE, Clarke MC. Cell death, damage-associated molecular patterns, and sterile inflammation in cardiovascular disease. Arterioscler Thromb Vasc Biol. 2011;31:2781-6.

3. Manning AS, Hearse DJ. Reperfusion-induced arrhythmias: mechanisms and prevention. J Mol Cell Cardiol. 1984;16:497-518.

4. Bolli R, Marbán E. Molecular and cellular mechanisms of myocardial stunning. Physiol Rev. 1999;79:609-34. 